\title{
Compton cooling in the afterglows of gamma-ray bursts: Application to GRB 980923 and GRB 971214
}

\author{
C.-I. Björnsson \\ Stockholm Observatory, S-133 36, Sweden \\ bjornsson@astro.su.se
}

\begin{abstract}
The role of Compton cooling in the standard model for the afterglows of gamma-ray bursts is considered. When electrons cool by scattering off their own synchrotron photons, three cooling regimes are identified in which the observed synchrotron radiation exhibits qualitatively different characteristics. Depending on the values of the source parameters, an afterglow may evolve through one, two or all three of these regimes. Since synchrotron radiation can be regarded as Compton scattering of the virtual photons due to the magnetic field, in one of these regimes the instantaneous synchrotron spectrum has properties identical to those when Compton cooling is negligible. During this phase in the evolution, the synchrotron radiation falls mainly in the near infrared/optical/UV spectral range. In order to break this degeneracy, good temporal coverage is needed. Alternatively, the importance of Compton scattering can be determined for those afterglows which are observed outside this degeneracy phase. It is suggested that the afterglows of GRB 980923 and GRB 971214 are two such cases. In the prompt afterglow of GRB 980923, the Klein-Nishina limit suppresses Compton scattering and the cooling is due to synchrotron radiation. However, the derived values of the source parameters are such that Compton cooling is expected to have been important in its subsequent evolution. The observed properties of GRB 971214 indicate cooling to be dominated by Compton scattering rather than synchrotron radiation. If Compton cooling is generally important in the afterglows of gamma-ray bursts, the likelihood of the shock becoming radiative is increased. It is suggested that this effect contributes to the low frequency of detected afterglows.
\end{abstract}

Subject headings: gamma rays:bursts — radiation mechanisms:nonthermal—shock waves

\section{Introduction}

In view of its relative simplicity, the standard model for the afterglows of gamma-ray bursts (Mészáros and Rees 1997; Sari et al. 1998) has proven to be surprisingly successful (e.g., Wijers et al. 1997; Galama et al. 1998). Synchrotron radiation from behind a spherically symmetric shock, caused 
by the interaction between an external medium and a relativistically expanding fireball, accounts for an impressive amount of observed characteristics. Deviations from the expected behavior observed for some afterglows during their later stages have been attributed to an aspherical outflow, for example, a jet geometry. Jet-edge effects and the lateral expansion of the outflow then increase the rate of decline of the light curves as well as the typical synchrotron frequency (Roads 1997; Harrison et al. 1999). However, afterglows exist for which the later stages of the evolution show no signs of deviation from a spherical outflow (e.g., Fruchter et al. 1999).

Kulkarni et al. (1999) noticed several afterglows to have soft X-ray fluxes in excess of that obtained by extrapolating from the infrared/optical spectral range. Such an additional spectral component is not expected and indicates that the standard model is incomplete. There are also some afterglows whose observed properties in the infrared/optical spectral range do not comply with standard predictions (e.g., Wijers and Galama 1999). Although the properties of most observed afterglows are well accommodated within the standard model, the reason is not clear for the small likelihood of a given gamma-ray burst to produce an observed afterglow; for example, an adiabatic outflow requires rather large variations in the source parameters between different gamma-ray bursts in order to suppress the emitted radiation below the observed values for the majority of the afterglows. This issue has been discussed by Akerlof et al. (2000) for the prompt afterglows. We suggest these shortcomings to be due, at least in part, to the neglect of Compton cooling. In order not to increase the number of free parameters in the standard model, it is assumed that Compton scattering occurs on the self-produced synchrotron photons.

Normally, arguments used for not considering Compton scattering together with synchrotron radiation include: The observations are well explained by synchrotron cooling only and energy equipartition between relativistic electrons and magnetic fields makes Compton scattering marginally important, at most, for the observed spectral evolution of the afterglows (Sari et al. 1996; Sari and Piran 1999; Moderski et al. 2000). For many afterglows, observations are not extensive enough to allow a determination of independent values for the source parameters and, hence, the assumption of equipartition is one of convenience, since the plasma properties behind the shock are not understood well enough to strongly motivate such a choice. However, sometimes the preferred value for the partition of energy between electrons and magnetic field is such that a consistent description requires Compton scattering to be included (e.g., Chevalier and Li 1999; Vreeswijk et al. 1999). This is in line with the arguments of Galama et al. (1999) that a major reason for the varied appearance of afterglows is a wide range of values for the magnetic field. The effects on the synchrotron spectrum from first order Compton scattering have been discussed by (Panaitescu and Kumar 2000; Sari and Ensin 2000; Wei and Lu 2000).

Synchrotron radiation can be seen as Compton scattering of virtual photons due to the magnetic field. Hence, in a situation when all electrons scatter off the same real photons, the instantaneous synchrotron spectrum is indistinguishable from one for which the cooling is due to synchrotron radiation (see also Sari and Ensin 2000). The main aim of the present paper is threefold: (i) Determine the phase of the afterglow evolution during which this spectral degeneracy 
occurs. (ii) Characterize the spectral properties and their evolution outside this degenerate phase for situations when Compton cooling affects the afterglow. (iii) Apply these results to observed afterglows in order to estimate the role played by Compton scattering.

There are two suggested physical settings for the gamma-ray burst phenomenon, namely, the merger of a neutron star with another compact object and the explosion of a hypernova (Paczyński 1998). For the afterglow, the main difference between these two scenarios is the assumed properties of the external medium into which the shock moves. In the former case, the external medium is assumed to have properties similar to the interstellar medium, while in the latter case the shock encounters a Wolf-Rayet-type stellar wind. The importance of Compton scattering is determined mainly by the value of the energy density in electrons relative that in magnetic fields. Since this ratio is likely due to local plasma processes behind the shock, rather than global properties, the inclusion of Compton scattering is qualitatively similar for the two cases. Hence, for convenience, the discussion is limited to the case with an interstellar-type external medium.

In $\S 2$ the relevant timescales are defined for afterglows in which the cooling is dominated by Compton scattering. It is shown that the evolution can contain up to three phases each with its own cooling characteristics. The spectral properties, including light curves, of these phases are discussed in $\S 3$. Two specific afterglows (GRB 980923 and GRB 971214) are discussed in $\S 4$. They have

been chosen since their observed properties suggest them to be outside the degenerate phase. The values derived for the source parameters imply Compton cooling to have significantly affected the evolution of both these afterglows. The implications for afterglows in general are briefly discussed in $\S 5$.

\section{Cooling behind the shock: Compton scattering versus synchrotron radiation}

In the standard model for the afterglow of gamma-ray bursts, electrons and magnetic fields are injected through a relativistic shock. There is evidence for a non-spherical shock; however, as long as the Lorentz factor of the shock is larger than the inverse of the angular extent of the asphericity, the observed evolution is well described by a spherically symmetric shock. The calculations below assume the validity of such a description. Furthermore, the discussion is restricted to the forward shock.

\subsection{The importance of Compton cooling}

We will adopt the usual convention of measuring the thermodynamic quantities of the shocked gas, including the energy density of photons, in its own restframe, while other quantities are measured in the restframe of the external medium. Let the electron energy distribution, injected behind the spherically symmetric shock, be given by $N(\gamma) \propto \gamma^{-p}$ for $\gamma \geq \gamma_{m}$ and zero otherwise, where $\gamma$ is the Lorentz factor of the electron. The typical frequency emitted by an electron with 
Lorentz factor $\gamma_{m}$ is $\nu_{m}$ and $\nu_{c}$ is the frequency emitted by those electrons $\left(\gamma_{c}\right)$, which have a cooling time equal to the dynamical time scale of the shock. In order to simplify the discussion, $2<p<3$ is assumed so that the peak of the energy density in synchrotron photons occurs at a frequency $\nu \approx \max \left(\nu_{m}, \nu_{c}\right)$.

When cooling is complete, the energy density of photons behind a relativistic shock is roughly equal to the energy density injected through the shock. If cooling is not complete (i.e., $\gamma_{c}>\gamma_{m}$ ), the photon energy density is smaller by a factor $\approx\left(\gamma_{c} / \gamma_{m}\right)^{2-p}$. Therefore, the energy density of synchrotron photons, $U_{\text {synch }}$, at $\nu_{c}$ is given by

$$
\frac{U_{\text {synch }}\left(\nu_{c}\right)}{B^{2} / 8 \pi} \approx \frac{\epsilon_{e}}{\epsilon_{B}}\left(\frac{\gamma_{c}}{\gamma_{m}}\right)^{2-p}
$$

where $\epsilon_{e}$ and $\epsilon_{B}$ are the fractions of the injected energy which go into relativistic electrons and magnetic fields $(B)$, respectively. The modification to equation (1), needed when Compton scattering off the self-produced synchrotron photons dominates the cooling, can be obtained as follows. Since synchrotron radiation can be regarded as Compton scattering of virtual photons due to the magnetic field ('zeroth' order Compton scattering), $U_{\text {synch }}(\nu) \approx \gamma^{2} \tau(\gamma) B^{2} / 8 \pi$, where $\tau(\gamma)$ is the Thomson optical depth of electrons, with Lorentz factor $\sim \gamma$, radiating at $\nu$. Likewise, when Compton scattering dominates the cooling, the main cooling of electrons with Lorentz factor $\gamma$ occurs on photons with frequency $\nu_{\gamma}$ with a corresponding energy density $U_{C o m p}\left(\nu_{\gamma}\right)$. Therefore, the analogue of equation (1), when Compton scattering dominates synchrotron radiation as the main cooling mechanism, takes the form

$$
\frac{U_{\text {synch }}\left(\nu_{c}\right)}{B^{2} / 8 \pi} \frac{U_{C o m p}\left(\nu_{\gamma_{c}}\right)}{B^{2} / 8 \pi} \approx \frac{\epsilon_{e}}{\epsilon_{B}}\left(\frac{\gamma_{c}}{\gamma_{m}}\right)^{2-p} .
$$

The relation given in equation (2) is a formal one and the expressions for $U_{C o m p}$ and $\nu_{\gamma}$ have to be determined for each situation. There are two simple, limiting cases; scattering in the KleinNishina limit which implies $\nu_{\gamma} \propto \gamma^{-1}$ and scattering in the Thomson limit when $\nu_{\gamma}$ is independent of $\gamma$ and all electrons cool on the same photons. The latter case can arise, for example, when $\gamma_{m}$ is large enough for the emission in first order Compton scattering to be well separated from the synchrotron component while, at the same time, the Klein-Nishina limit prevents appreciable radiation in second order Compton scattering. In such a situation $U_{C o m p}=U_{\text {synch }}$ and $\nu_{\gamma}=\max \left(\nu_{m}, \nu_{c}\right)$ and equation (2) reduces to

$$
\frac{U_{\text {synch }}\left(\nu_{c}\right)}{B^{2} / 8 \pi} \approx\left[\frac{\epsilon_{e}}{\epsilon_{B}}\left(\frac{\gamma_{c}}{\gamma_{m}}\right)^{2-p}\right]^{\frac{1}{2}} .
$$

Cooling due to scattering in the Thomson limit is expected to be similar to synchrotron cooling, since, as mentioned above, synchrotron radiation can be regarded as 'zeroth' order Compton scattering on virtual photons, which are the same for all electrons independent of their energy. On the 
other hand, cooling in the Klein-Nishina limit should exhibit characteristics quite different from those of either synchrotron cooling or scattering in the Thomson limit. We now discuss and contrast the salient features of the observed radiation in these three limits, i.e., cooling due to synchrotron radiation, Compton scattering, respectively, in the Thomson and Klein-Nishina limits.

\subsection{Characteristics of afterglows when cooling is dominated by Compton scattering}

The above arguments show that Compton cooling is important whenever

$$
\frac{\epsilon_{e}}{\epsilon_{B}} \geq \zeta \max \left[1,\left(\frac{\nu_{c}}{\nu_{m}}\right)^{\frac{p-2}{2}}\right] .
$$

The value of $\zeta$ is model dependent but $\zeta \sim v / c$ is expected, where $v$ is the velocity of the shock relative the shocked gas. Hence, as mentioned above, for a relativistic shock $\zeta$ is of order unity. When source parameters are such that the condition in equation (4) is fulfilled, Compton scattering should affect the evolutionary properties of the afterglow. It is convenient to divide the observed behavior into three phases according to the different cooling regimes. Synchrotron cooling dominates early on, while Compton scattering in the Thomson limit can take over during an intermediate stage. For $\gamma_{m}$ small enough, multiple Compton orders become possible. Hence, at late times multiple scattering may be possible in which cooling is likely to occur in the Klein-Nishina limit. Furthermore, cooling in the Klein-Nishina limit is expected to be important also during the transition between the different phases. These separate phases will be referred to as the synchrotron, Thomson, and multiple Compton scattering phase, respectively. As is shown below, in the standard scenario for the afterglow, its emission may go through one, two, or all three of these phases.

The scattering cross-section for an electron with Lorentz factor $\gamma$ drops rapidly for photon frequencies larger than $\nu_{K N}(\gamma)$ (the Klein-Nishina limit), given by

$$
\gamma \frac{\nu_{K N}(\gamma)}{\Gamma} \approx \frac{m c^{2}}{h}
$$

where $\Gamma$ is the bulk Lorentz factor of the shocked gas, $m$ is the electron mass, and $h$ is the Planck constant. Following Sari et al. (1998), we use $\gamma_{m}=6.1 \times 10^{2} \epsilon_{e} \Gamma$, which corresponds to $p=2.5$ and define $\eta \equiv \gamma / \gamma_{m}$ or, equivalently, $\nu \equiv \eta^{2} \nu_{m}$, which leads to

$$
\nu_{K N}(\eta) \approx \frac{2.0 \times 10^{17}}{\epsilon_{e} \eta}
$$

The expressions for $\nu_{m}$ and $\nu_{s c}$, the cooling frequency for negligible Compton losses, can be written as

$$
\nu_{m}=1.2 \times 10^{15} E_{52}^{1 / 2} t_{d}^{-3 / 2} \epsilon_{e}^{2} \epsilon_{B}^{1 / 2}
$$

and

$$
\nu_{s c}=2.9 \times 10^{12} E_{52}^{-1 / 2} n^{-1} t_{d}^{-1 / 2} \epsilon_{B}^{-3 / 2},
$$


where $E_{52}$ is the total energy of the shocked gas in units of $10^{52} \mathrm{ergs}, t_{d}$ is the observed time, in days, since the onset of the expansion, and $n$ is the number density of the external medium. Furthermore, $\Gamma=6.7\left(E_{52} / n\right)^{1 / 8} t_{d}^{-3 / 8}$ (Wijers and Galama 1999, henceforth WG99) has been used in equations (7) and (8). The cooling frequency, including the effects of Compton scattering, can then be written

$$
\nu_{c}=\frac{\nu_{s c}}{\left[1+U_{C o m p}\left(\nu_{\gamma_{c}}\right) /\left(B^{2} / 8 \pi\right)\right]^{2}} .
$$

The time dependence of these frequencies can be used to determine the characteristic times when the salient features of the Compton scattering change. The properties of the observed synchrotron radiation depend sensitively on the relative values of $\nu_{m}$ and $\nu_{c}$. Consider the case when scattering occurs in the Thomson limit and let $t_{e q}$ denote the time when $\nu_{m}=\nu_{c}$. With the use of $U_{C o m p}\left(\nu_{\gamma_{c}}\right) \approx U_{\text {synch }}\left(\nu_{c}\right) \approx\left(\epsilon_{e} / \epsilon_{B}\right)^{1 / 2}\left(B^{2} / 8 \pi\right)$ in equation (9), one finds for $t_{e q}$, in days,

$$
t_{d, e q}=4.1 \times 10^{2} E_{52} n \epsilon_{e}^{3} \epsilon_{B} .
$$

With the use of equation (3), the time dependence of $\eta_{c} \equiv\left(\nu_{c} / \nu_{m}\right)^{1 / 2}$ can then be written

$$
\eta_{c}=\left(\frac{t}{t_{e q}}\right)^{1 / 2} \max \left\{1,\left(\frac{t}{t_{e q}}\right)^{(p-2) /[2(4-p)]}\right\} .
$$

Note that this expression for $\eta_{c}$ is generally valid for Compton scattering in the Thomson limit, i.e., it is not restricted to the case when $t=t_{e q}$ falls in the Thomson phase.

Assume that $\eta_{c}<1$ at the beginning of the Thomson phase. An electron with Lorentz factor $\gamma$ scatters in the Thomson limit when $\nu_{K N}(\eta)>\nu_{m}$. From equations (6) and (7) this leads to

$$
t(\eta)>t_{1 C} \eta^{2 / 3}
$$

where the value of $t_{1 C}$, in days, is

$$
t_{d, 1 C}=3.3 \times 10^{-2} E_{52}^{1 / 3} \epsilon_{e}^{2} \epsilon_{B}^{1 / 3} .
$$

Hence, $t_{1 C}$ marks the transition from the synchrotron to the Thomson phase and the condition $\eta_{c}<1$ at the transition is equivalent to $t_{e q}>t_{1 C}$. If instead $\eta_{c}>1$ at the beginning of the Thomson phase, the condition for scattering in the Thomson limit becomes $\nu_{K N}(\eta)>\nu_{c}$ or

$$
t(\eta)>t_{1 C}\left(\frac{\eta}{\eta_{c}}\right)^{2 / 3} \eta_{c}^{2}
$$

However, since $\eta_{c}^{2}$ increases more rapidly with time than linearly in the Thomson phase for $\eta_{c}>1$ $(2<p<3)$, equation (14) leads to a contradiction, since $\eta \geq \eta_{c}$ for cooling to be important. As a result, $t_{e q}<t_{1 C}$ implies that no transition to the Thomson phase takes place and the source remains in the synchrotron phase during the entire evolution. The reason is that the value of $\gamma_{c}$ increases with time so fast that the Klein-Nishina limit suppresses efficient cooling due to Compton scattering. 
The condition for the source to remain in the Thomson phase for $t>t_{e q}$ (i.e., $\eta_{c}>1$ ) is

$$
\gamma_{c}^{2} \nu_{c}>\nu_{K N}(\eta)
$$

This leads to

$$
t(\eta)<t_{2 C}\left(\frac{\eta}{\eta_{c}}\right)^{4 / 9} \eta_{c}^{20 / 9}
$$

where the value of $t_{2 C}$, in days, is

$$
t_{d, 2 C}=1.6 \times 10^{2} E_{52}^{1 / 3} n^{-1 / 9} \epsilon_{e}^{20 / 9} \epsilon_{B}^{2 / 9} .
$$

Again, if the condition in equation (16) is valid at $t=t_{e q}$, it remains so for all times, since $\eta_{c}^{20 / 9}$ increases more rapidly with time than linearly. Hence, for $t_{e q}<t_{2 C}$, no transition occurs to the multiple Compton scattering phase. In order for such a transition to be possible, $t_{e q}>t_{2 C}$ is required, in which case the transition takes place at $t=t_{2 C}$ (cf. eq. [16] with $\eta_{c}=1$ ).

When there is no transition to the multiple Compton scattering phase, the source remains in the Thomson phase as long as $\nu_{K N}\left(\eta_{c}\right)>\nu_{c}$, which implies

$$
t<t_{e q}\left(\frac{t_{e q}}{t_{1 C}}\right)^{(4-p) /(p-2)} .
$$

Furthermore, in order for Compton cooling to be important the inequality in equation (4) must be valid, which results in $(\zeta=1)$

$$
t<t_{e q}\left(\frac{\epsilon_{e}}{\epsilon_{B}}\right)^{(4-p) /(p-2)} .
$$

Hence, for $t_{1 C}<t_{e q}<t_{2 C}$, the duration of the Thomson phase is determined by the relative values of $t_{e q} / t_{1 C}$ and $\epsilon_{e} / \epsilon_{B}$. These characteristic times are useful for describing the properties of the emitted radiation only as long as $t \lesssim t_{d}(\Gamma=1)$, where $t_{d}(\Gamma=1)=1.6 \times 10^{2}\left(E_{52} / n\right)^{1 / 3}$, in days, is the approximate time when the outflow becomes subrelativistic.

The values of $t_{1 C}$ and $t_{2 C}$ correspond to the times when those electrons, which provide the main contribution to the photon energy density, are able to scatter their own radiation into the next higher Compton order (cf. eqs. [12] and [16]). However, as mentioned above, cooling in the next higher Compton order may become important before these transition times. The scattering then occurs in the Klein-Nishina limit on frequencies below that where the photon energy density peaks. The extent of these transition stages depends on the relative strength of the Compton orders (i.e., $\left.\epsilon_{e} / \epsilon_{B}\right)$.

With the use of equations (10), (13), and (17) the relation $t_{1 C}<t_{e q}<t_{2 C}$ can be written

$$
8.0 \times 10^{-5}<E_{52}^{2 / 3} n \epsilon_{e} \epsilon_{B}^{2 / 3}<3.4 \times 10^{-1} n^{-1 / 9} \epsilon_{e}^{2 / 9} \epsilon_{B}^{-1 / 9}
$$

An alternative way of writing this relation is

$$
7.2 \times 10^{-7} E_{52}^{-1} n^{-3 / 2} \epsilon_{e}^{-5 / 2}<\epsilon_{B} / \epsilon_{e}<2.5 \times 10^{-1} E_{52}^{-6 / 7} n^{-10 / 7} \epsilon_{e}^{-2} .
$$


The three cooling regimes in Figure 1 are delimited by the three characteristic times $t_{1 C}, t_{2 C}$, and $t_{e q}$. Energy requirements suggest a value of $\epsilon_{e}$ not much smaller than unity. Hence, since $t_{e q}$ is more sensitive than $t_{1 C}$ and $t_{2 C}$ to variations in $E_{52}$ and $n$ (cf. eqs. [10], [13], and [17]), the change which occurs for values of $E_{52}$ and $n$ other than the ones used in Figure $1\left(E_{52}=10\right.$ and $n=1)$ is due mainly to the variation in $t_{e q}\left(\propto E_{52} n\right)$. It is seen from equation (21) and Figure 1 that a significant multiple Compton scattering phase can occur during the later stages of the afterglow for large values of $E_{52}$ and/or $n$. Likewise, small values of $E_{52}$ and $n$ limit the extent of the Thomson phase or can exclude it altogether for small values of $\epsilon_{B} / \epsilon_{e}$. Although $\epsilon_{B} / \epsilon_{e} \ll 1$ for such cases, synchrotron radiation dominates the cooling due to the Klein-Nishina limit in the scattering cross-section.

In summary (see Figure 1 ), the value of $t_{e q}$ relative to $t_{1 C}$ and $t_{2 C}$ determines the salient features of the Compton scattered radiation. If Compton cooling significantly affects the observed emission, a rough characterization of the source evolution can be done as follows.

$t_{e q}<t_{1 C}$ : Synchrotron phase. Initially, cooling is dominated by synchrotron radiation. Later on first order Compton scattering in the Klein-Nishina limit may become important but no transition to the Thomson phase takes place.

$t_{1 C}<t_{e q}<t_{2 C}$ : Synchrotron and Thomson phases. Like the previous case but here a transition occurs to the Thomson phase at $t \approx t_{1 C}$. A transition back to the synchrotron phase takes place for values of $t_{e q}$ close to $t_{1 C}$ (cf. eq. [18]), while values of $t_{e q}$ close to $t_{2 C}$ can cause second order Compton scattering in the Klein-Nishina limit to become important later on.

$t_{2 C}<t_{e q}$ : Synchrotron, Thomson, and multiple Compton scattering phases. Like the previous case except that at $t \approx t_{2 C}$ multiple Compton scatterings become possible. Cooling is then likely to occur in the Klein-Nishina limit.

\section{The emitted synchrotron spectrum when Compton cooling is important}

Depending on the values of the source parameters (cf. eq. [20] or [21]), the Compton scattering can evolve through one, two, or all three of the phases discussed above. The observed characteristics of the resulting synchrotron radiation are quite different in the various phases and we now consider them in turn.

\subsection{The synchrotron phase}

In the beginning of this phase, cooling is dominated by synchrotron radiation. At some later time, depending on the value of $\epsilon_{B} / \epsilon_{e}$, first order Compton scattering in the Klein-Nishina limit may take over as the main cooling agent. For this latter stage of the synchrotron phase it is convenient to introduce a parameter $\eta_{\text {crit }}$; it corresponds to the normalized Lorentz factor of those electrons, which can Compton scatter on their own synchrotron photons in the Klein-Nishina limit 
(cf. eq. [6]),

$$
\eta_{\text {crit }}=\left(\frac{2.0 \times 10^{17}}{\epsilon_{e} \nu_{m}}\right)^{1 / 3} .
$$

Consider first the case $\eta_{\text {crit }}<1$. This condition holds during the whole synchrotron phase for $t_{e q}>t_{1 C}$. Assuming no cooling at $\nu_{m}$ leads to $U_{C o m p}\left(\nu_{\eta}\right) \propto \eta^{-4 / 3}$ for $\eta \geq 1$. This shows the Compton cooling time scale to decrease with decreasing electron energy. Hence, if significant Compton cooling occurs in the synchrotron phase for $\eta_{\text {crit }}<1$, electrons radiating at $\nu_{m}$ are affected. For such situations the spectral shape below $\nu_{m}$ is important. The minimum value of $\eta$, for which the main Compton cooling occurs on photons below $\nu_{m}$ is obtained from $\nu_{K N}\left(\eta_{\min }\right) \equiv \nu_{m}$ or

$$
\eta_{\min }=\frac{2.0 \times 10^{17}}{\epsilon_{e} \nu_{m}}=\eta_{c r i t}^{3}
$$

The corresponding synchrotron frequency is

$$
\nu_{\min }=\eta_{\min }^{2} \nu_{m}=\eta_{c r i t}^{6} \nu_{m}
$$

The electrons which cool in the Klein-Nishina limit on frequencies around $\nu_{\min }$ have a normalized Lorentz factor, $\eta_{\max }$, determined from $\nu_{K N}\left(\eta_{\max }\right) \equiv \nu_{\min }$ or

$$
\eta_{\max }=\frac{2.0 \times 10^{17}}{\epsilon_{e} \nu_{\min }}=\eta_{\text {crit }}^{-3} .
$$

The corresponding synchrotron frequency is

$$
\nu_{\max }=\eta_{\max }^{2} \nu_{m}=\eta_{\text {crit }}^{-6} \nu_{m} .
$$

Hence, electrons with $\eta_{c r i t}^{3}<\eta<\eta_{\text {crit }}^{-3}$ cool on photons in the frequency range $\nu_{m} \eta_{c r i t}^{6}<\nu<\nu_{m}$. If Compton cooling is important for these electrons, their energy distribution can be obtained in a self-consistent way.

Let $N(\eta) \propto \eta^{-p_{c}}$ for $\eta_{c r i t}^{3}<\eta<1$. In the corresponding frequency range, the spectral flux, $F_{\nu} \propto \nu^{-\alpha}$, has $\alpha=\left(p_{c}-1\right) / 2$. Furthermore, steady injection of electrons with $\eta \geq 1$ implies $N(\eta) \propto-(d \eta / d t)^{-1}$. Since it is assumed that Compton scattering dominates the cooling $d \eta / d t \propto-\eta^{2} U_{C o m p}\left(\nu_{\eta}\right) \propto-\eta^{\left(p_{c}+1\right) / 2}$, where $\nu_{\eta} \propto \eta^{-1}$. Equating these two independent expressions for $N(\eta)$ yields $\eta^{p_{c}}=\eta^{\left(p_{c}+1\right) / 2}$, which implies $p_{c}=1$ and, hence, $\alpha=0$ in the frequency range $\eta_{\text {crit }}^{6}<\nu / \nu_{m}<1$. Furthermore, electrons radiating in the frequency range $1<\nu / \nu_{m}<\eta_{\text {crit }}^{-6}$ suffer cooling at a rate $d \eta / d t \propto-\eta$. This results in an exponentially decreasing energy with time, i.e., cooling time is independent of electron energy. As a consequence, in the latter frequency range, cooling does not affect the spectral index, i.e., $\alpha=\alpha_{o} \equiv(p-1) / 2$. Hence, the spectral break at $\eta_{\text {crit }}^{-6} \nu_{m}$ could be mistaken for a cooling break. However, since $\eta_{c r i t}^{-6} \propto \nu_{m}^{2} \propto t^{-3}$, such a spectral break would decrease with time much faster than any cooling frequency. The actual cooling break occurs below $\eta_{\text {crit }}^{6} \nu_{m}$.

Although of less interest for the present paper, the rest of the spectrum can be determined in a similar manner (Fig. 2). Electrons radiating in the frequency range below $\eta_{c r i t}^{6} \nu_{m}$ cool on photons 
with frequencies larger than $\nu_{m}$. Unless $\eta_{\text {crit }}$ is close to unity, cooling of these electrons occurs on photons in the $\alpha_{o}$ part of the spectrum. This results in a steepening of the spectrum below $\eta_{c r i t}^{6} \nu_{m}$ from $\alpha=0$ to $\alpha=\alpha_{o} / 2$. The high energy electrons, which cool on photons in the $\alpha_{o} / 2$ part of the spectrum, radiate at frequencies larger than $\eta_{c r i t}^{-6} \nu_{m}$. With the same reasoning as above, this causes a steepening of the spectrum from $\alpha=\alpha_{o}$ to $\alpha=(5 / 4) \alpha_{o}$. At even higher frequencies, the radiating electrons cool on the low frequency, $F_{\nu} \propto \nu^{1 / 3}$, part of the synchrotron spectrum. This cooling, if it is important, results in a flattening of the spectrum and the spectral index is given by $\alpha=\alpha_{o}-1 / 6$. At these high frequencies, synchrotron cooling can become important even if $\epsilon_{e} \gg \epsilon_{B}$ because the effective value of $U_{\text {Comp }}$ decreases rapidly with increasing $\gamma$.

In the synchrotron phase, $\eta_{c r i t}>1$ occurs for $t_{e q}<t_{1 C}$ and implies $\nu_{c}>\nu_{m}$. Electrons with $\eta_{\text {crit }}^{-3}<\eta<\eta_{\text {crit }}^{3}$ have $\nu_{m}<\nu_{K N}(\eta)<\eta_{\text {crit }}^{6} \nu_{m}$. If Compton cooling is important and $\nu_{c}<\eta_{\text {crit }}^{6} \nu_{m}$, $\nu_{K N}\left(\eta_{c}\right)>\nu_{m}$ is implied, which results in $U_{C o m p}\left(\nu_{\eta_{c}}\right) /\left(B^{2} / 8 \pi\right) \propto \nu_{m}^{(p-1) / 8} \nu_{c}^{(p-5) / 8}$ (cf. eq. [2]). Inserting this in equation (9) yields $\nu_{c} \propto t^{(3 p-7) /[2(p-1)]}$. The observed value of $p$ is such that $\nu_{c}$ should increase with time for most afterglows. An even faster increase of $\nu_{c}$ with time occurs for $\nu_{c}>\eta_{c r i t}^{6} \nu_{m}$, since then the cooling takes place on the $U_{\text {synch }} \propto \nu^{4 / 3}$ part of the spectrum.

The above discussion shows that the spectrum in the synchrotron phase is expected to consist of a rather large number of spectral ranges with different spectral indices. The finite spectral width of the emission from the individual electrons causes a gradual transition from one spectral range to another. Unless a given spectral range is rather wide, the spectral indices will vary continuously with frequency and the expressions derived above should be regarded as a rough guide to the typical values expected in the different parts of the spectrum. Furthermore, this smoothing of the spectral features is increased by the finite escape time of the photons from behind the relativistic shock.

\subsection{The Thomson phase}

The Thomson phase occurs for $t_{e q}>t_{1 C}$ and begins at $t \approx t_{1 C}$, which corresponds to

$$
\begin{aligned}
\nu_{m} & =2.0 \times 10^{17} \epsilon_{e}^{-1} \\
\nu_{c} & =1.6 \times 10^{13} E_{52}^{-2 / 3} n^{-1} \epsilon_{e}^{-2} \epsilon_{B}^{-2 / 3}\left(=\nu_{m} \frac{t_{1 C}}{t_{e q}}\right) .
\end{aligned}
$$

In this phase the Klein-Nishina limit plays a minor role, except preventing significant amount of second order Compton scattering. Since all the electrons cool on the same photons, i.e., $\nu_{\gamma}=$ $\max \left(\nu_{m}, \nu_{c}\right)$ does not depend on $\gamma$, the behavior of the synchrotron flux is expected to be similar to the case when only synchrotron radiation contributes to the cooling. The main differences which arise from using $U_{C o m p}$ instead of $B^{2} / 8 \pi$ in the cooling rates, concern the magnitude and time dependence of $\nu_{c}$ (see also Panaitescu and Kumar 2000).

As shown in $\S 2.2$ (see also Fig. 1), $t<t_{e q}$ in the Thomson phase for $t_{e q}>t_{2 C}$. For such conditions, the light curves during the Thomson phase are the same as those obtained for negligible 
cooling due to Compton scattering (the synchrotron case). The only difference is the value of $\nu_{c}$, which is smaller by a factor $\epsilon_{B} / \epsilon_{e}$ as compared to the value in the synchrotron case. The situation changes for $t_{e q}<t_{2 C}$, since then $t=t_{e q}$ occurs during the Thomson phase and the steeper time dependence of $U_{C o m p}$ for $t>t_{e q}$ causes the rate at which $\nu_{c}$ declines to slow down. Since $\nu_{m} \propto t^{-3 / 2}$, the value of $\nu_{c}$ even increases for $p>8 / 3$ (cf. eq. [11]). The change in the time dependence of $\nu_{c}$ takes place when $\nu_{c}=\nu_{m} \equiv \nu_{e q}$, where

$$
\nu_{e q}=1.4 \times 10^{11} E_{52}^{-1} n^{-3 / 2} \epsilon_{e}^{-5 / 2} \epsilon_{B}^{-1} .
$$

This causes the light curves to differ from the synchrotron case for $t>t_{e q}$. Let the light curves be characterized by $\beta$ such that the flux $F_{\nu} \propto t^{-\beta}$. In the synchrotron case, there is a break in the light curves as $\nu_{c}\left(>\nu_{m}\right)$ passes through a given frequency band $\left(\nu<\nu_{e q}\right)$ and $\beta$ increases by $1 / 4$ from $3(p-1) / 4$ to $(3 p-2) / 4$ (Sari et al. 1998). Since $F_{\nu} \propto \nu_{c}^{1 / 2}$ for $\nu>\nu_{c}$, equation (11) shows this break to be less abrupt in the Thomson phase; for $p<8 / 3$, the value of $\beta$ increases only by $1 / 4-(p-2) /[2(4-p)]=(8-3 p) /[4(4-p)]$. In addition to this chromatic break, there is also an achromatic break occurring at $t=t_{e q}$ in the light curves for $\nu>\nu_{e q}$, which is absent in the synchrotron case. Again using the fact that $F_{\nu} \propto \nu_{c}^{1 / 2}$ for $\nu>\nu_{c}$ together with equation (11) result in a decrease of $\beta$ by (p-2)/[2(4-p)] from its earlier value (3p-2)/4, i.e., this break corresponds to a flattening of the light curves (Fig. 3a). The value of $\nu_{c}$ increases with time for $p>8 / 3$ and $t>t_{e q}$. In such situations, there is no break in the light curves for $\nu<\nu_{e q}$, instead there are two breaks for $\nu>\nu_{e q}$; first comes the achromatic break and then the chromatic one (Fig. 3b). As was mentioned in $\S 1$, the instantaneous synchrotron spectral properties in the Thomson phase should be identical to those in the synchrotron case. It is seen here that this is also true for the light curves when $t<t_{e q}$.

The solution for the source parameters in terms of the observables in the synchrotron case was given by WG99. The corresponding solution in the Thomson phase can be obtained in a similar manner (cf. Sari and Ensin 2000). In the Thomson phase one can write for $\nu_{c}>\nu_{m}$

$$
\begin{aligned}
F_{\nu_{m}, \text { norm }} & =E_{52} n^{1 / 2} \epsilon_{e}^{1 / 2} \\
\nu_{m, \text { norm }} & =E_{52}^{1 / 2} \epsilon_{e}^{2} \epsilon_{B}^{1 / 2} \\
\nu_{c, \text { norm }} & =E_{52}^{-1 / 2} n^{-1} \epsilon_{e}^{-1} \epsilon_{B}^{-1 / 2}\left(\frac{\nu_{c}}{\nu_{m}}\right)^{(p-2) / 2} \\
\nu_{a, \text { norm }} & =E_{52}^{1 / 5} n^{3 / 5} \epsilon_{e}^{-1} \epsilon_{B}^{1 / 5}
\end{aligned}
$$

where $F_{\nu_{m}, \text { norm }}, \nu_{m, \text { norm }}, \nu_{c, \text { norm }}$, and $\nu_{a, \text { norm }}$ are the normalized expressions, respectively, for $F_{\nu_{m}}$, $\nu_{m}, \nu_{c}$, and the synchrotron self-absorption frequency $\nu_{a}$. The normalization is the same as used by WG99. From equations (30a) - (30d), the source parameters can be expressed in terms of the observables as

$$
E_{52}=F_{\nu_{m}, \text { norm }}^{2} \nu_{m, \text { norm }}^{-3 / 2} \nu_{c, \text { norm }}^{-1 / 2} \nu_{a, \text { norm }}^{-5 / 2}\left(\frac{\nu_{c}}{\nu_{m}}\right)^{(p-2) / 4}
$$




$$
\begin{aligned}
n & =\nu_{m, \text { norm }}^{-7 / 6} \nu_{c, \text { norm }}^{-3 / 2} \nu_{a, \text { norm }}^{-5 / 6}\left(\frac{\nu_{c}}{\nu_{m}}\right)^{3(p-2) / 4} \\
\epsilon_{e} & =\nu_{m, \text { norm }}^{-1 / 6} \nu_{c, \text { norm }}^{-1 / 2} \nu_{a, \text { norm }}^{-5 / 6}\left(\frac{\nu_{c}}{\nu_{m}}\right)^{(p-2) / 4} \\
\epsilon_{B} & =F_{\nu_{m}, \text { norm }}^{-2} \nu_{m, \text { norm }}^{25 / 6} \nu_{c, \text { norm }}^{5 / 2} \nu_{a, \text { norm }}^{35 / 6}\left(\frac{\nu_{c}}{\nu_{m}}\right)^{-5(p-2) / 4} .
\end{aligned}
$$

In order for the solution in equation (31a) $-(31 \mathrm{~d})$ to be consistent, $\epsilon_{e} / \epsilon_{B}>\zeta\left(\nu_{c} / \nu_{m}\right)^{(p-2) / 2}$. From equations (31c) and (31d) this requirement is equivalent to

$$
\left(\frac{\nu_{c}}{\nu_{m}}\right)^{p-2}>\zeta F_{\nu_{m}, \text { norm }}^{-2} \nu_{m, \text { norm }}^{13 / 3} \nu_{c, \text { norm }}^{3} \nu_{a, \text { norm }}^{20 / 3} .
$$

A consistent solution for the synchrotron case requires $\epsilon_{e} / \epsilon_{B}<\zeta\left(\nu_{c} / \nu_{m}\right)^{(p-2) / 2}$. With the use of the equations in WG99, one finds

$$
\left(\frac{\nu_{c}}{\nu_{m}}\right)^{p-2}>\zeta^{-2} F_{\nu_{m}, \text { norm }}^{-2} \nu_{m, \text { norm }}^{13 / 3} \nu_{c, \text { norm }}^{3} \nu_{a, \text { norm }}^{20 / 3}
$$

Except for the different $\zeta$-dependence, the consistency requirement is the same for the Thomson phase and synchrotron case. Since $\zeta \sim 1$ for a relativistic shock, this shows that for the afterglows of gamma-ray bursts, the two solutions cannot be distinguished from each other when only the synchrotron component of the spectrum is observed. It is straightforward to show that this conclusion remains valid also for the stage when $\nu_{c}<\nu_{m}$. The degeneracy between these two solutions will be broken if the derived source parameters do not comply with the requirements discussed in $\S 2.2$ for being in the Thomson phase.

\subsection{The multiple Compton scattering phase}

The multiple Compton scattering phase occurs for $t_{e q}>t_{2 C}$ and begins at $t \approx t_{2 C}$, which corresponds to

$$
\begin{aligned}
& \nu_{m}=5.7 \times 10^{11} n^{1 / 6} \epsilon_{e}^{-4 / 3} \epsilon_{B}^{1 / 6} \\
& \nu_{c}=2.3 \times 10^{11} E_{52}^{-2 / 3} n^{-17 / 18} \epsilon_{e}^{-19 / 9} \epsilon_{B}^{-11 / 18}\left(=\nu_{m} \frac{t_{2 C}}{t_{e q}}\right) .
\end{aligned}
$$

For parameters typical of the afterglows of gamma-ray bursts, at these late times the different Compton orders are expected to overlap; hence, the scattered flux forms a continuum in which the different orders are not easily distinguished. The electrons then cools in the Klein-Nishina limit, i.e., $\nu_{\gamma}=\nu_{K N}(\gamma)$. The requirement that the total energy of the shock should not be excessive suggests that the value of $\epsilon_{e}$ is not much smaller than unity. Equations (34) and (35) then show that the synchrotron radiation in the multiple Compton scattering phase is likely to fall mainly in the far infrared/radio spectral range. The properties of the observed flux under such conditions are discussed by Björnsson and Aslaksen (2000). 


\section{Discussion}

The best studied afterglows have been observed mainly in the near infrared/optical spectral range. For values of source parameters thought relevant for the standard model, this corresponds to the frequency range where the synchrotron radiation is expected to be emitted during the Thomson phase (cf. eqs. [27] and [34]). In this phase, it is hard to distinguish observationally between two very different physical situations; namely, one in which Compton scattering dominates the cooling and another where Compton scattering plays a minor role. In fact, a rather large parameter space exists (cf. eq. [20] or [21]) in which it is not even in principle possible to separate the two cases when only the synchrotron component is observed (cf. Sari and Ensin 2000).

In this section the afterglows of GRB 980923 and GRB 971214 are discussed. They have been selected primarily because their observed characteristics suggest that they do not entirely radiate in the phase where the values of the source parameters are degenerate. Hence, these afterglows should allow a determination of the importance of Compton scattering in their evolution. The afterglow of GRB 980923 is likely to be in the synchrotron phase. The rather odd behavior of the infrared/optical afterglow in GRB 971214 (eg., WG99) is ascribed to the source being in the transition stage between the Thomson and multiple Compton scattering phases.

\subsection{GRB 980923}

The BATSE observations of the prompt afterglow from GRB 980923 exhibited a break in the spectrum which evolved with time towards lower frequencies (Giblin et al. 1999). Interpreted as synchrotron radiation from the forward shock, Giblin et al. (1999) showed this break to be consistent with the synchrotron cooling frequency, $\nu_{s c}$, and incompatible with $\nu_{m}$. Their conclusion is based on two independent pieces of evidence, namely the time evolution of the break frequency as well as the flux. However, as shown in $\S 3.2$, synchrotron radiation and Compton scattering in the Thomson phase have similar cooling characteristics. Since $\nu_{c}>\nu_{m}$, the cooling frequency evolves slower with time in the Thomson phase than in the synchrotron case. The observed value $p=2.4$ implies $\nu_{c} \propto t^{1 / 4}$ (cf. eq. [11]) instead of $\nu_{s c} \propto t^{1 / 2}$. Although the observed light curve favors synchrotron cooling, the uncertainties in both the flux and spectral slope are such that Compton scattering in the Thomson phase cannot be excluded. We therefore consider both cases together.

It is convenient to introduce the parameter $\epsilon_{e, c r i t} \equiv \zeta \epsilon_{B}\left(\nu_{c} / \nu_{m}\right)^{(p-2) / 2}$; this is the value of $\epsilon_{e}$ for which cooling due to synchrotron radiation equals that in Compton scattering (cf. eq. [4]). The observed fluence $(\mathcal{F})$ can then be related to the source parameters as

$$
\begin{aligned}
\mathcal{F} & =\frac{\epsilon_{B} E(1+z)}{4 \pi d^{2}} \frac{\epsilon_{e}}{\epsilon_{e, \text { crit }}}, \\
\mathcal{F} & =\frac{\epsilon_{B} E(1+z)}{4 \pi d^{2}}\left(\frac{\epsilon_{e}}{\epsilon_{e, \text { crit }}}\right)^{1 / 2},
\end{aligned}
$$


where equations (36a) and (36b) correspond, respectively, to cooling due to synchrotron radiation and Compton scattering in the Thomson phase. The lower fluence in the Thomson phase is due to the fact that only a fraction $\left(\epsilon_{e, c r i t} / \epsilon_{e}\right)^{1 / 2}$ of the emitted energy emerges as synchrotron radiation (cf. eq. [3]). The luminosity distance to the source is denoted by $d$. Likewise, expressions for $\epsilon_{B}$ are obtained from equations (8) and (9). With the use of the observed value $\nu_{c}=3.0 \times 10^{19} \mathrm{~Hz}$ at $t=40 \mathrm{~s}$, one finds

$$
\begin{aligned}
& \epsilon_{B}=2.7 \times 10^{-4} n^{-2 / 3} E_{52}^{-1 / 3}(1+z)^{-1 / 3} \\
& \epsilon_{B}=2.7 \times 10^{-4} n^{-2 / 3} E_{52}^{-1 / 3}(1+z)^{-1 / 3}\left(\frac{\epsilon_{e, \text { crit }}}{\epsilon_{e}}\right)^{2 / 3},
\end{aligned}
$$

for the two cases. The fluence in the afterglow for $t>40 \mathrm{~s}$ is $\approx 3.0 \times 10^{-5} \mathrm{ergs} \mathrm{cm}^{-2}$, which results in

$$
\begin{aligned}
& E_{52}=1.6 \times 10^{6} n(1+z)^{-1} d_{28}^{3}\left(\frac{\epsilon_{e, \text { crit }}}{\epsilon_{e}}\right)^{3 / 2} \\
& E_{52}=1.6 \times 10^{6} n(1+z)^{-1} d_{28}^{3}\left(\frac{\epsilon_{e}}{\epsilon_{e, \text { crit }}}\right)^{1 / 4}
\end{aligned}
$$

where $d_{28}$ is the luminosity distance in units of $10^{28} \mathrm{~cm}$. It is seen from equations (38a) and (38b) that observations require $E_{52}>1.6 \times 10^{6} n(1+z)^{-1} d_{28}^{3}$ for both the synchrotron $\left(\epsilon_{e}<\epsilon_{e, \text { crit }}\right)$ and Thomson $\left(\epsilon_{e}>\epsilon_{e, \text { crit }}\right)$ case. For standard values of $E_{52}$ and $n$, this places the source in the local universe (i.e., $d_{28} \ll 1$ ). Since the distance to GRB 980923 is not known such a solution cannot be ruled out a priori. The identical constraints on the source parameters obtained for the synchrotron case and Compton scattering in the Thomson phase is an example of the general result derived in $\S 3.2$.

A cosmological distance can be made compatible with standard values for $E_{52}$ and $n$ if the afterglow of GRB 980923 is in the synchrotron phase and $\epsilon_{e} \gg \epsilon_{e, c r i t}$. Assuming the afterglow to be in the transition stage between the synchrotron and Thomson phases implies that the cooling is due to first order Compton scattering in the Klein-Nishina limit. However, as discussed in $\S 3.1$, possible spectral breaks under such conditions either increase with time $(p=2.4)$ or decrease too rapidly with time to be compatible with the evolution of the observed spectral break. Hence, a consistent description requires the cooling to be due to synchrotron radiation.

The requirement of negligible Compton cooling at $\nu_{s c}$ constrains the allowed values of the source parameters. For a given observed flux at $\nu_{s c}$, the value of $U_{C o m p}\left(\nu_{\eta_{s c}}\right)$ increases with $\eta_{c r i t}$; hence, the weakest constraints are obtained for $\eta_{\text {crit }}<1$. Since no low frequency break in the spectrum is apparent, $\nu_{m}<6.0 \times 10^{18} \mathrm{~Hz}$. The expression for $\eta_{\text {crit }}$ (eq. [22]) together with $\eta_{\text {crit }}<1$ then requires $\epsilon_{e}>1 / 30$. If Compton cooling is important at $\nu_{m}$, the electrons would cool down quickly to at least $\eta_{\text {min }}=\eta_{c r i t}^{3}$, which results in a flat $(\alpha=0)$ spectrum in the frequency range $\eta_{c r i t}^{6} \leq \nu / \nu_{m} \leq 1$ (cf. $\S 3.1$ ). This increase in flux below $\nu_{m}$ makes the cooling time independent of frequency in the range $\eta_{c r i t}^{6} \leq \nu / \nu_{m} \leq \eta_{\text {crit }}^{-6}$. Hence, assuming $\nu_{s c}<\eta_{c r i t}^{-6} \nu_{m}$, no Compton cooling at $\nu_{s c}$ is equivalent to no Compton cooling at $\nu_{m}$. 
Negligible Compton cooling at $\nu_{m}$ requires

$$
\nu_{m}<\frac{\nu_{s c}}{\left[U_{\text {Comp }}\left(\nu_{\gamma_{m}}\right) /\left(B^{2} / 8 \pi\right)\right]^{2}} .
$$

Since the energy density below $\nu_{m}$ is proportional to $\nu^{4 / 3}, U_{C o m p}\left(\nu_{\gamma_{m}}\right)=\eta_{\text {crit }}^{4} U_{\text {synch }}\left(\nu_{m}\right)$. Furthermore, $U_{\text {synch }}\left(\nu_{m}\right) /\left(B^{2} / 8 \pi\right)=\left(\epsilon_{e} / \epsilon_{B}\right)\left(\nu_{m} / \nu_{s c}\right)^{1 / 2}$ and equation (39) yields

$$
\frac{\epsilon_{B}}{\epsilon_{e}}>\eta_{c r i t}^{4} \frac{\nu_{m}}{\nu_{s c}}
$$

With the use of equation $(22)$ and $\nu_{s c}=3.0 \times 10^{19}$ this can be written

$$
\epsilon_{B}>6.7 \times 10^{-3} \eta_{\text {crit }} .
$$

When $\nu_{s c}>\eta_{c r i t}^{-6} \nu_{m}$, the restrictions set by the lack of Compton scattering is more model dependent. However, a definite lower limit to $\epsilon_{B}$ can be obtained by assuming negligible Compton cooling only at $\nu_{s c}$ but (unphysically) no enhancement of the flux below $\nu_{m}$ due to cooling of electrons with $\eta<\eta_{s c}$. The condition $U_{C o m p}\left(\nu_{\eta_{s c}}\right)<B^{2} / 8 \pi$ then leads to

$$
\frac{\epsilon_{B}}{\epsilon_{e}}>\eta_{c r i t}^{4}\left(\frac{\nu_{m}}{\nu_{s c}}\right)^{7 / 6}
$$

This lowers the limit of $\epsilon_{B}$ in equation (40) only marginally. With the use of the expression for $\eta_{\text {crit }}$ (eq. [22]) and the observed upper limit of $\nu_{m}$, one finds from equation (42)

$$
\epsilon_{B}>1.6 \times 10^{-3} \epsilon_{e}^{-1 / 3}
$$

or a factor 1.3 larger if equation (40) is used.

Although the main aim of the present paper is to discuss the qualitative features introduced by significant Compton cooling in an otherwise standard synchrotron model, it should be mentioned that the minimum values of $\epsilon_{B}$ in equations (40) and (42) are likely to be slightly too restrictive for two reasons. The above estimate assumes $\zeta=1$. A lower value may be appropriate, since even for a relativistic shock $v=c / 3$. Furthermore, the value used for the Klein-Nishina frequency is too large; the decline in the cross-section sets in at somewhat lower frequencies than assumed in equation (5) (Blumenthal and Gould 1970). For example, using $\zeta=1 / 3$ and a value of the KleinNishina frequency half as large as that in equation (5) reduce the lower limit of $\epsilon_{B}$ by a factor 7.6 as compared to those given above.

With the assumption of a cosmological distance to GRB 980923, the observations of its afterglow together with equations (37a) and (38a) indicate: (1) A value of $E_{52}$ substantially larger than unity and/or a value of $n$ substantially smaller than unity, although within the range thought applicable for the standard scenario. (2) The value of $\epsilon_{B}$ needs to be close to its lower limit allowed by the observed lack of Compton cooling $\left(\sim 10^{-3}\right)$. (3) The value of $\epsilon_{e}$ cannot be much smaller 
than unity. This shows that in order for the afterglow to be detected by BATSE, the values of $\epsilon_{e}$ and $\epsilon_{B}$ are quite constrained. For values of $\epsilon_{B}$ considerably smaller than given in equation (43) Compton cooling dominates and for values larger than this, $\nu_{s c}$ falls below the BATSE frequency range; in either case, the result is a diminishing observed fluence. Likewise, a value of $\epsilon_{e}$ considerably smaller than unity also lowers the fluence below the detectability of BATSE. Hence, in general, for canonical values of $E$ and $n$, the detection of a prompt afterglow by BATSE implies values of $\epsilon_{B}$ and $\epsilon_{e}$ not too different from the ones derived above for GRB 980923.

Sari (1997) pointed out that for large values of $\epsilon_{e}$ the shock may become radiative if $\nu_{c} \leq \nu_{m}$. He showed that the energy in the shock decreases, roughly, as $E(t)=E_{o}\left(t / t_{o}\right)^{-\epsilon_{e}}$. In the case of GRB 980923, $\nu_{c} \geq \nu_{m}$ for most of the synchrotron phase. With the parameters derived above $t_{e q}>t_{1 C}$ (cf. eq. [20] or [21]) and, hence, the afterglow should enter the Thomson phase, which implies $t_{o} \sim t_{1 C}$. The cooling of the shock in the Thomson phase continues until $t \sim t_{e q}$. The fraction of the initial shock energy which is radiated away is then $1-\left(t_{1 C} / t_{e q}\right)^{\epsilon_{e}}$. The rather large value deduced above for $\epsilon_{e}$ suggests that radiative cooling may have been important for the dynamics of the afterglow of GRB 980923 in its later phases.

\subsection{GRB 971214}

The afterglow of GRB 971214 showed a drop in the K-band flux by a factor $3-4$ between $t\left(=t_{1}\right)=0.20$ days and $t\left(=t_{2}\right)=0.58$ days (Gorosabel et al. 1998). At $t_{2}$ the spectrum exhibited a break at $3 \times 10^{14} \mathrm{~Hz}$ (Kulkarni et al. 1998; Ramaprakash et al. 1998) together with evidence for significant reddening (Halpern et al. 1998). Within the context of a standard synchrotron model, this optical break can be due either to $\nu_{m}$ or $\nu_{s c}$. However, WG99 emphasized that either choice gives rise to serious inconsistencies. Interpreted as $\nu_{m}$, the break predicts an increasing flux in the K-band between $t_{1}$ and $t_{2}$ rather than the observed decrease. WG99 used the synchrotron model and its expected correlation between spectral index and rate of decline of the light curve to correct for the reddening. Assuming that the break corresponds to $\nu_{s c}$, and $\nu_{m}<\nu_{s c}$, in order to account for the decline of K-band flux, the dereddening leads to a flat or weakly declining intrinsic flux distribution below the break. This is in contrast to the predicted rise corresponding to a spectral index $(p-1) / 2$. WG99 also discussed several other unattractive features, which result from a straightforward application of the synchrotron model.

Interpreting the optical break as $\nu_{s c}$ leaves the X-ray flux unexplained, while it is accounted for when the break is due to $\nu_{m}$. However, the latter case strains the derived source parameters; for example, in order to be a consistent solution, $\epsilon_{e} / \epsilon_{B} \lesssim\left(\nu_{s c} / \nu_{m}\right)^{(p-2) / 2}$ (cf. eq. [4]). The observed values $\nu_{s c} / \nu_{m} \gtrsim 5 \times 10^{3}$ and $p=2.7$ together with $\epsilon_{e} \lesssim 1$, imply $n \lesssim 2 \times 10^{-3}$. This upper limit to the density is much smaller than values expected in the standard model. Hence, even disregarding the initial K-band flux measurements, the observed spectrum at $t_{2}$ makes the synchrotron model incomplete. 
In view of the limitations of the synchrotron model to account for the observed properties of the afterglow in GRB 971214, we suggest the discrepancy between observations and model predictions to be due to the neglect of Compton scattering. Below we discuss a scenario in which the observations correspond to the stage when the dominant contribution to the cooling changes from first to second order Compton scattering. The infrared/optical emission is synchrotron radiation, while the Xray emission is due to Compton scattering of the lower frequency synchrotron radiation. The optical spectral break is assumed to be due to $\nu_{m}$, which, in turn, is larger than $\nu_{c}$, i.e., cooling is important for all electrons injected at the shockfront. At $t_{1}$ second order Compton scattering starts to dominate the cooling at $\nu_{K}\left(\geq \nu_{c}\right)$, the frequency corresponding to the K-band. For larger frequencies cooling is still in the Thomson limit. Hence, the spectral index between $\nu_{K}$ and $\nu_{m}$ is $1 / 2$. At $t_{2}$ second order Compton scattering dominates the cooling in the frequency range below $\nu_{m}$. It is assumed that $\nu_{K N}\left(\gamma_{K}\right)$ lies on the $F_{\nu} \propto \nu^{1 / 3}$ part of the first order Compton component. Hence, $U_{C o m p}\left(\nu_{\gamma}\right) \propto \gamma^{-4 / 3}$, which leads to a spectral index of $-1 / 6$ between $\nu_{K}$ and $\nu_{m}$ (cf. $\S 3.1$ ).

With the use of $\nu_{m} \propto t^{-3 / 2}$ and $F_{\nu_{m}} \propto t^{1 / 2}\left(t \lesssim t_{2}\right)$, the fluxes at $\nu_{K}$ at $t_{1}$ and $t_{2}$, respectively, are related by

$$
\frac{F_{\nu_{K}}\left(t_{2}\right)}{F_{\nu_{K}}\left(t_{1}\right)}=\left(\frac{\nu_{K}}{\nu_{m}\left(t_{2}\right)}\right)^{2 / 3}\left(\frac{t_{1}}{t_{2}}\right)^{1 / 4}
$$

Since $\nu_{m}\left(t_{2}\right) / \nu_{K} \sim 3$ and $t_{2} / t_{1} \sim 3$, equation (44) yields $F_{\nu_{K}}\left(t_{1}\right) / F_{\nu_{K}}\left(t_{2}\right) \sim 3$, which is consistent with the observed value. Hence, the proposed scenario can account for the decline in the K-band flux as well as the spectral index below the break at $t_{2}$. We now discuss the values of the source parameters which result from the above interpretation of the observations. The aim is to show that such a scenario is plausible rather than to perform a detailed comparison. In order to achieve the latter, numerical calculations are necessary; in particular, since second order Compton scattering is assumed to play an important role.

Including the redshift dependence in equation (7), the observed values of $\nu_{m}\left(t_{2}\right)=3.0 \times 10^{14}$ and $z=3.42$ lead to

$$
E_{52} \epsilon_{B} \epsilon_{e}^{4}=2.8 \times 10^{-3}
$$

With the use of equation (45) one finds in the Thomson phase

$$
\frac{\nu_{c}}{\nu_{m}}=4.0 \times 10^{-2} \frac{\epsilon_{e}}{n} \frac{t}{t_{1}}
$$

It is shown below that a consistent solution suggests a value of $\epsilon_{e} / n$ not substantially below unity. The time dependence of $\nu_{m}$ implies $\nu_{m}\left(t_{1}\right) \approx 15 \nu_{K}$. Hence, $\nu_{c}$ in equation (46) is close to $\nu_{K}$ at $t_{1}$ and second order Compton scattering affects the cooling only for $t \gtrsim t_{1}$. Furthermore, the K-band flux should reach a maximum for $\nu_{c} \approx \nu_{K}$, i.e., at $t \approx t_{1}$. The observations of Gorosabel et al. (1998) are consistent with this expectation; although the errors are rather large, the flux measured at $t=0.15$ is roughly equal to that at $t_{1}(=0.20)$, i.e., the decline in the K-band flux is likely to have started at $t \approx t_{1}$. 
The flux at $\nu_{m}$ in the Thomson phase for $\nu_{c}<\nu_{m}$ is

$$
F_{\nu_{m}}(t)=1.2 \frac{h_{70}^{2}}{(\sqrt{1+z}-1)^{2}} E_{52} n^{1 / 2} \epsilon_{B}^{1 / 2}\left(\frac{\nu_{c}}{\nu_{m}}\right)^{1 / 2} \quad \text { mJy }
$$

where the numerical coefficient is taken from WG99. For simplicity, the cosmological parameters have been chosen as $\Omega=1$ and $\Lambda=0$ and $h_{70}$ is the Hubble constant normalized to $70 \mathrm{~km} \mathrm{~s}^{-1}$ $\mathrm{Mpc}^{-1}$. Since it is assumed that second order Compton scattering affects the cooling at $\nu_{m}$ only for $t \gtrsim t_{2}$, equation (47) can be used together with the reddening corrected (WG99) value $F_{\nu_{m}}\left(t_{2}\right)=30$ $\mu \mathrm{Jy}$ to deduce

$$
E_{52}=3.2 \epsilon_{e}^{3}
$$

or from equation (45)

$$
\epsilon_{B} \epsilon_{e}^{7}=8.8 \times 10^{-4}
$$

where $h_{70}=1$ has been used.

During the Thomson phase, the synchrotron energy density peaks around $\nu_{m}$ (for $\nu_{c}<\nu_{m}$ ), while the energy density of first order Compton scattered radiation reaches a maximum around $\gamma_{m}^{2} \nu_{m}$. Hence, equation (3) implies

$$
\nu F_{\nu}\left(\nu=\nu_{m}\right) \sim\left(\frac{\epsilon_{B}}{\epsilon_{e}}\right)^{1 / 2} \nu F_{\nu}\left(\nu=\gamma_{m}^{2} \nu_{m}\right) .
$$

Since cooling at $t_{1}$ due to first and second order Compton scattering is assumed to be equal for electrons with Lorentz factors around $\gamma_{K}$, at this time the photon energy densities at $\nu_{m}$ and $\nu_{K N}\left(\gamma_{K}\right)$ should be roughly equal. Furthermore, in order for the Compton cooling below $\nu_{m}$ at $t_{2}$ to occur on the $\nu^{1 / 3}$ part of the first order Compton flux, $\nu_{K N}\left(\gamma_{K}\right)$ cannot be significantly larger than $\gamma_{c}^{2} \nu_{c}$ at $t_{1}$. Hence, an upper limit to $\epsilon_{B} / \epsilon_{e}$ is obtained from equation (50) by using $\nu_{K N}\left(\gamma_{K}\right) \sim \gamma_{c}^{2} \nu_{c}$

$$
\left(\frac{\epsilon_{B}}{\epsilon_{e}}\right)^{1 / 2} \lesssim\left(\frac{\gamma_{c}^{2} \nu_{c}}{\gamma_{m}^{2} \nu_{m}}\right)^{1 / 2} \sim \frac{\nu_{c}}{\nu_{m}}
$$

where logarithmic factors in the Compton scattered component have been neglected, i.e., a spectral index of $1 / 2$ is assumed between $\gamma_{c}^{2} \nu_{c}$ and $\gamma_{m}^{2} \nu_{m}$. From equations (46) and (49) this results in

$$
\frac{\epsilon_{e}^{5}}{n} \gtrsim 0.7
$$

The major uncertainty in the numerical value in equation (52) is likely due to the neglect of light travel time effects. The typical time for escape through the shockfront for a photon produced in the shock is the dynamical time scale. Inclusion of a finite escape time for the photons has two major consequences, both of which tend to decrease the lower limit in equation (52). Since cooling is important, higher frequencies are produced closer to the shock front than are the lower ones. Hence, at a given time, the observed radiation at lower frequencies reflects conditions at an 
earlier time than do that at larger frequencies. The rate of energy injection at the shock front decreases with time, which suggests the energy density in the first Compton component to increase less rapidly between $\gamma_{c}^{2} \nu_{c}$ and $\gamma_{m}^{2} \nu_{m}$ than assumed in equation (51). This expectation is consistent with the finding of Dal Fiume et al. (2000) that the X-ray spectral index as measured by BeppoSAX is likely to be larger than 0.5; their preferred value is 0.6. Furthermore, higher Compton orders involve photons which were originally produced at earlier times. Again, due to the decreasing rate of energy injection at the shockfront, at a given time the relative strength of higher Compton orders is increased for larger escape times. A quantitative evaluation of these effects requires numerical calculations. However, it is not likely that they will change the main conclusion of the above analysis; namely, in order for Compton scattering to give a consistent description of the afterglow of GRB 971214, the value of $\epsilon_{e}$ cannot be significantly below unity while that of $n$ cannot be much larger than unity. We note that the values deduced for the source parameters of GRB 971214 are rather similar to those for GRB 980923; in particular, the value of $\epsilon_{e}$ is quite large, which suggests that radiative cooling may be important.

The parameter values derived above relied on the assumption that the source was in the Thomson phase at $t_{1}$. At $t_{2}$ this is not so since the flat or slightly rising spectrum up to $\nu_{m}$ is ascribed to the effects of second order Compton scattering. As emphasized before, inclusion of multiple Compton scattering considerably increases the complexity of the problem; however, the spectrum at $t_{2}$ presented by WG99 can be used to make a independent consistency check of the scenario discussed above. For the redshift of GRB 971214, $\nu_{K N}\left(\gamma_{m}\right) \approx 10^{17} \mathrm{~Hz}$. Since cooling due to second order Compton scattering is assumed to be important below $\nu_{m}$ at that time, the energy densities at $\nu_{m}$ and $\nu_{K N}\left(\gamma_{m}\right)$ should be approximately equal. Observations show that $\nu F_{\nu}$ is roughly a factor of three larger at X-ray frequencies $\left(\approx 10^{18}\right)$ than at $\nu_{m}$. Although the spectral shape of the Compton scattered radiation is unknown below the X-ray band, this factor may be too small to account for the observed synchrotron spectrum below $\nu_{m}$. There are two effects, which can account for this possible difference. The reddening correction applied by WG99 is substantial. It was calculated assuming a pure synchrotron model. However, the reddening correction may be smaller, since the correlation between intrinsic spectral index and rate of decline of the flux, used by WG99, is no longer valid for the scenario discussed here. Furthermore, the scattered photons have on average a longer escape time than the synchrotron photons. For a given observed flux, this results in a larger effective energy density behind the shock of the former as compared to the latter.

\section{Conclusions}

In the standard model for the afterglows of gamma-ray bursts, the observed properties are determined by the values of four parameters $\left(E, n, \epsilon_{e}\right.$, and $\left.\epsilon_{B}\right)$. Plausible values of $E$ and $n$ come from the overall scenario for the gamma-ray burst phenomenon, while the values of $\epsilon_{e}$ and $\epsilon_{B}$ are most likely the result of not-so-well understood local plasma processes. Although energy requirements favor values of $\epsilon_{e}$ not substantially below unity, the value of $\epsilon_{B}$ is considerably harder 
to constrain. Equipartition arguments have been invoked to motivate the use of $\epsilon_{B} \sim \epsilon_{e}$.

Modelfitting of well observed afterglows can be used to derive values of the source parameters. However, for values of $E$ and $n$ thought relevant for the standard model, a large fraction of the afterglows are likely to have been observed during a phase in their evolution in which they suffer from a spectral degeneracy; namely, the observed spectra can be fitted equally well by cooling either due to synchrotron radiation or Compton scattering. Although, in principle, well sampled light curves can break this degeneracy, observations are usually not extensive enough to accomplish this. It was argued that the afterglows of GRB 980923 and GRB 971214 were observed outside this degeneracy phase. The derived source parameters indicate Compton cooling to have played an important role during their evolution and, hence, $\epsilon_{B} \ll \epsilon_{e}$ at least for these afterglows.

When cooling is enhanced by significant Compton scattering, a minor fraction of the radiated energy escapes as synchrotron emission. As a result, a given set of observations of the synchrotron component corresponds, on average, to larger values of $\epsilon_{e}$ and/or $E$ than for negligible Compton scattering. Although the accuracy of the values determined for the source parameters in the afterglows of GRB 980923 and GRB 971214 is rather low, it was emphasized that values of $\epsilon_{e}$ close to unity are implied for both of them. Together with the extended times during which the cooling was complete, this suggests the possibility of the shock becoming radiative. If cooling in the afterglows of most gamma-ray bursts is dominated by Compton scattering, the values of $\epsilon_{e}$ would be larger than normally deduced. Hence, for a radiative shock, rather small variations in the values of $\epsilon_{e}$ could lead to large changes in the emitted flux during the later stages of the evolution, for example, when the synchrotron emission falls in the infrared/optical spectral range. It was also pointed out that in order for prompt afterglows, like the one connected to GRB 980923, to be detected by BATSE the values of $\epsilon_{e}$ and $\epsilon_{B}$ are quite restricted.

This research was supported by a grant from the Swedish Natural Science Research Council. 


\section{REFERENCES}

Akerlof, C., et al. 2000, ApJ, 532, L25

Björnsson, C.-I. \& Aslaksen, T. 2000. ApJ, 533, 787

Blumenthal, G. R., \& Gould, R. J. 1970, Rev. Mod. Phys., 42, 237

Chevalier, R. A., \& Li, Z.-Y. 1999, ApJ, 520, L29

Dal Fiume, D., et al. 2000, A\&A, 355, 454

Fruchter, A. S., et al. 1999, ApJ, 516, 683

Galama, T. J., Wijers, R. A. M. J., Bremer, M., Groot, P. J., Strom, R. G., Kouveliotou, C., \& van Paradijs, J. 1998, ApJ, 500, L97

Galama, T. J., et al. 1999, Nature, 398, 394

Giblin, T. J., et al. 1999, ApJ, 524, L47

Gorosabel, J., 1998, A\&A, 335, L5

Halpern, J. P., Thorstensen, J. R., Helfand, D. J., \& Costa, E. 1998, Nature, 393, 41

Harrison, F. A., et al. 1999, ApJ, 523, L121

Kulkarni, S. R., et al. 1998, Nature, 393, 35

Kulkarni, S. R., et al. 1999, Nature, 398, 389

Mészáros, P., \& Rees, M. J. 1997, ApJ, 476, 232

Moderski, R., Sikora, M., \& Bulik, T. 2000, ApJ, 529, 151

Paczyński, B. 1998, ApJ, 494, L45

Panaitescu, A., \& Kumar, P. 2000, ApJ, 543, 66

Ramaprakash, A. N., et al. 1998, Nature, 393, 43

Roads, J. E. 1997, ApJ, 487, L1

Sari, R. 1997, ApJ, 489, L37

Sari, R., \& Ensin, A.A. 2001, ApJ, 548, 787

Sari, R., Narayan, R., \& Piran, T. 1996, ApJ, 473, 204

Sari, R., Piran, T., \& Narayan, R. 1998, ApJ, 497, L17 
Sari, R., \& Piran, T. 1999, ApJ, 520, 641

Vreeswijk, P. M., et al. 1999, ApJ, 523, 171

Wijers, R. A. M. J., Rees, M. J., \& Mészáros, P. 1997, MNRAS, 288, L51

Wei, D. M., \& Lu, T. 2000, A\&A, 360, L13

Wijers, R. A. M. J., \& Galama, T. J. 1999, ApJ, 523, 177 (WG99) 
Fig. 1. - The change of dominant cooling mode with time for $\epsilon_{B}<\epsilon_{e}$. The different cooling regimes are shown for $E_{52}=10, n=1, \epsilon_{e}=1 / 2$, and $p=5 / 2$ (synchrotron phase: light shading; Thomson phase: no shading; and multiple Compton scattering phase: dark shading). The change which occurs for other parameter values is due mainly to variations in $t_{e q}\left(\propto E_{52} n\right)$. The transition between different cooling modes is smooth due to Compton scattering in the Klein-Nishina limit (see text).

Fig. 2.- A schematic representation of the synchrotron spectrum in the synchrotron phase when cooling by Compton scattering in the Klein-Nishina limit is important for $\eta_{\text {crit }}<1$. The main features to note are the spectral indices around $\nu_{m}$; the one below $\nu_{m}$ is zero, while that in the region above $\nu_{m}$ is the same as for negligible cooling. For frequencies larger than $\nu_{1} \equiv \eta_{1}^{2} \nu_{m}$, cooling occurs on photons from the $\nu^{1 / 3}$-part of the spectrum. Effects due to synchrotron cooling and synchrotron self-absorption are not included. The spectral breaks between the different powerlaw regimes would be smooth in a real spectrum.

Fig. 3. - Light curves in the Thomson phase. Only those breaks are shown which are not present for negligible Compton cooling. The break which occurs at $t=t_{e q}$ for $\nu>\nu_{e q}$ is achromatic, while the time for the break taking place at $t>t_{e q}$ is frequency dependent. Panel (a) corresponds to $2<p<8 / 3$ and panel (b) to $8 / 3<p<3$. 


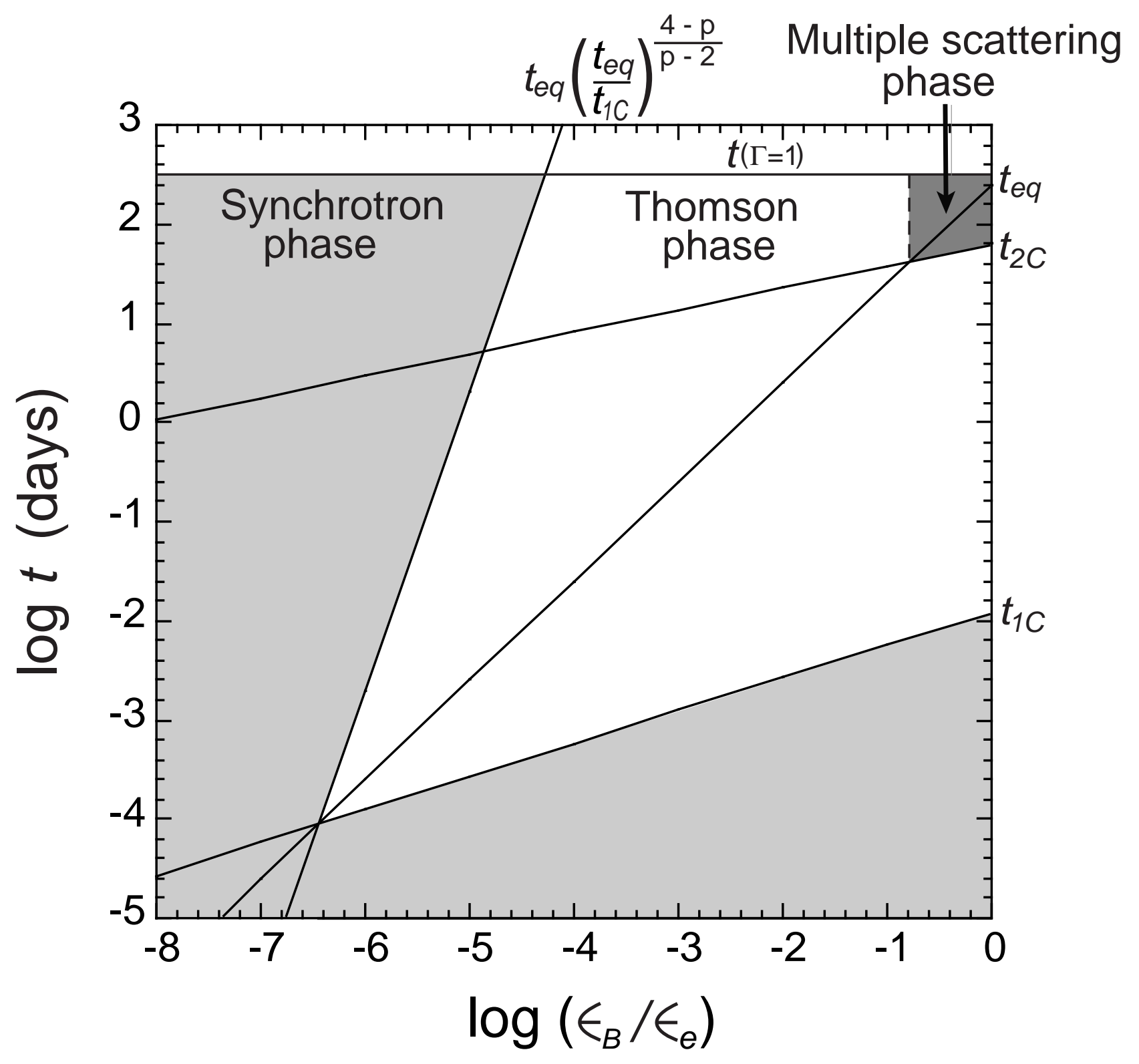




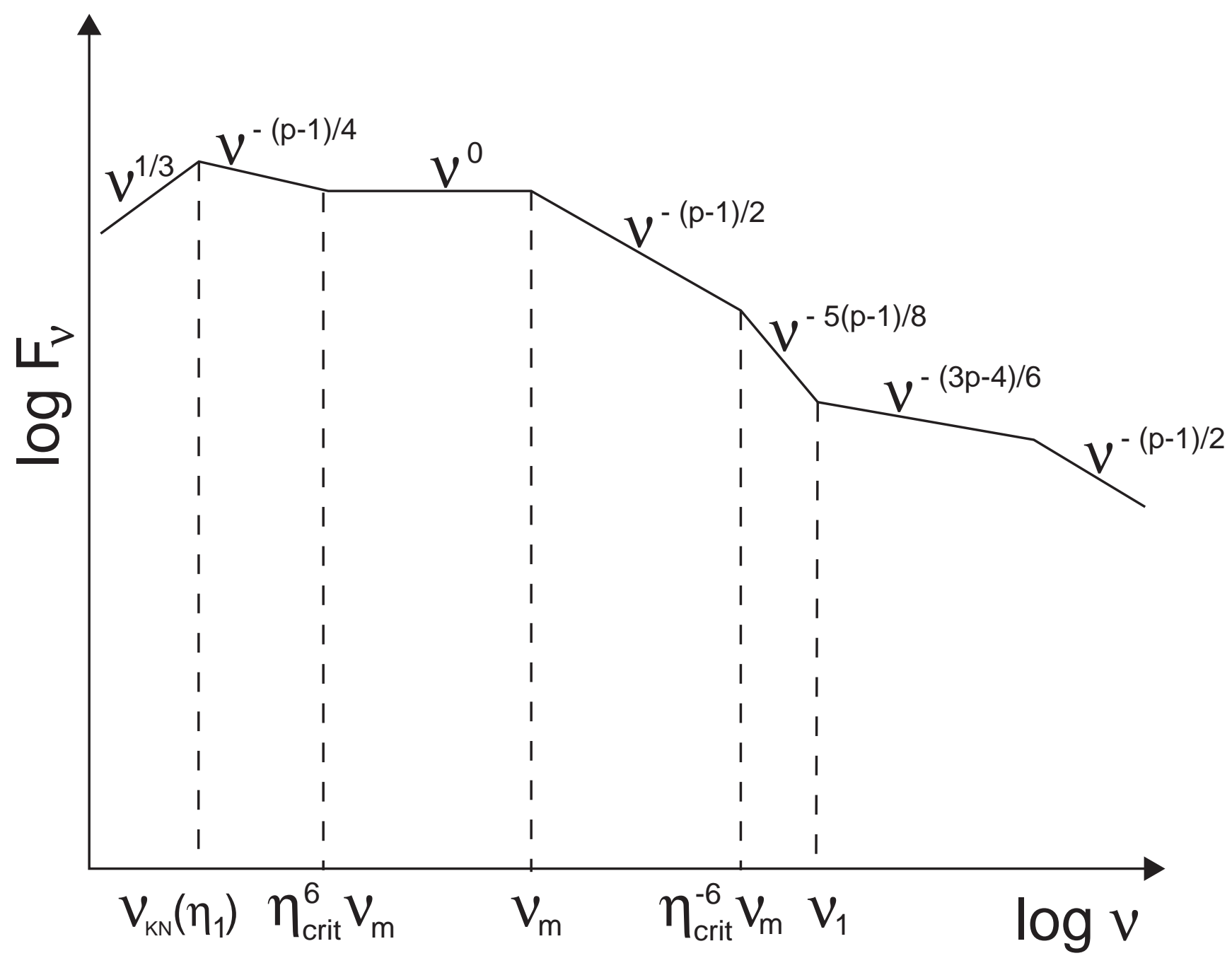


(a) $2<$ p $<8 / 3$

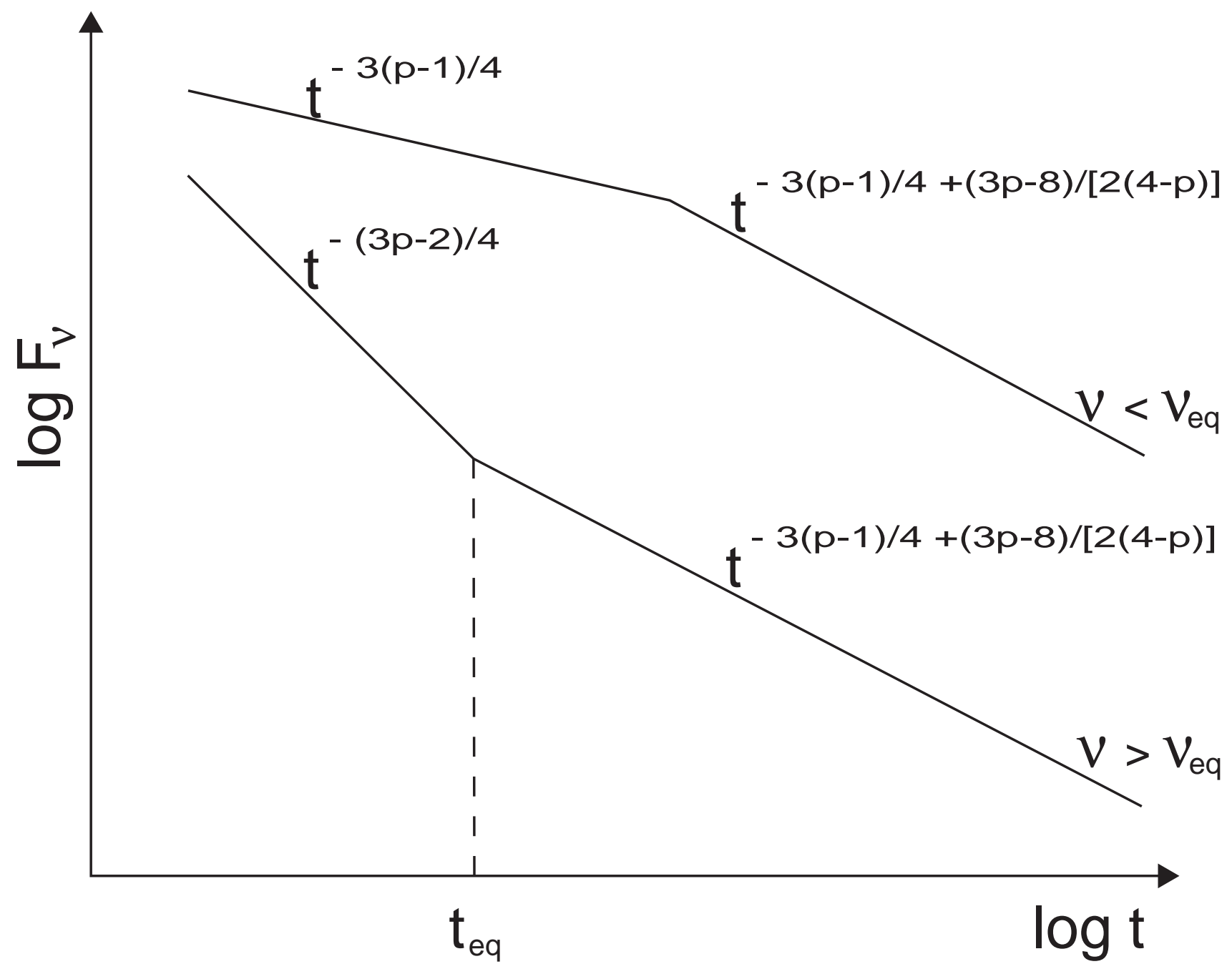


(b) $8 / 3<$ p $<3$

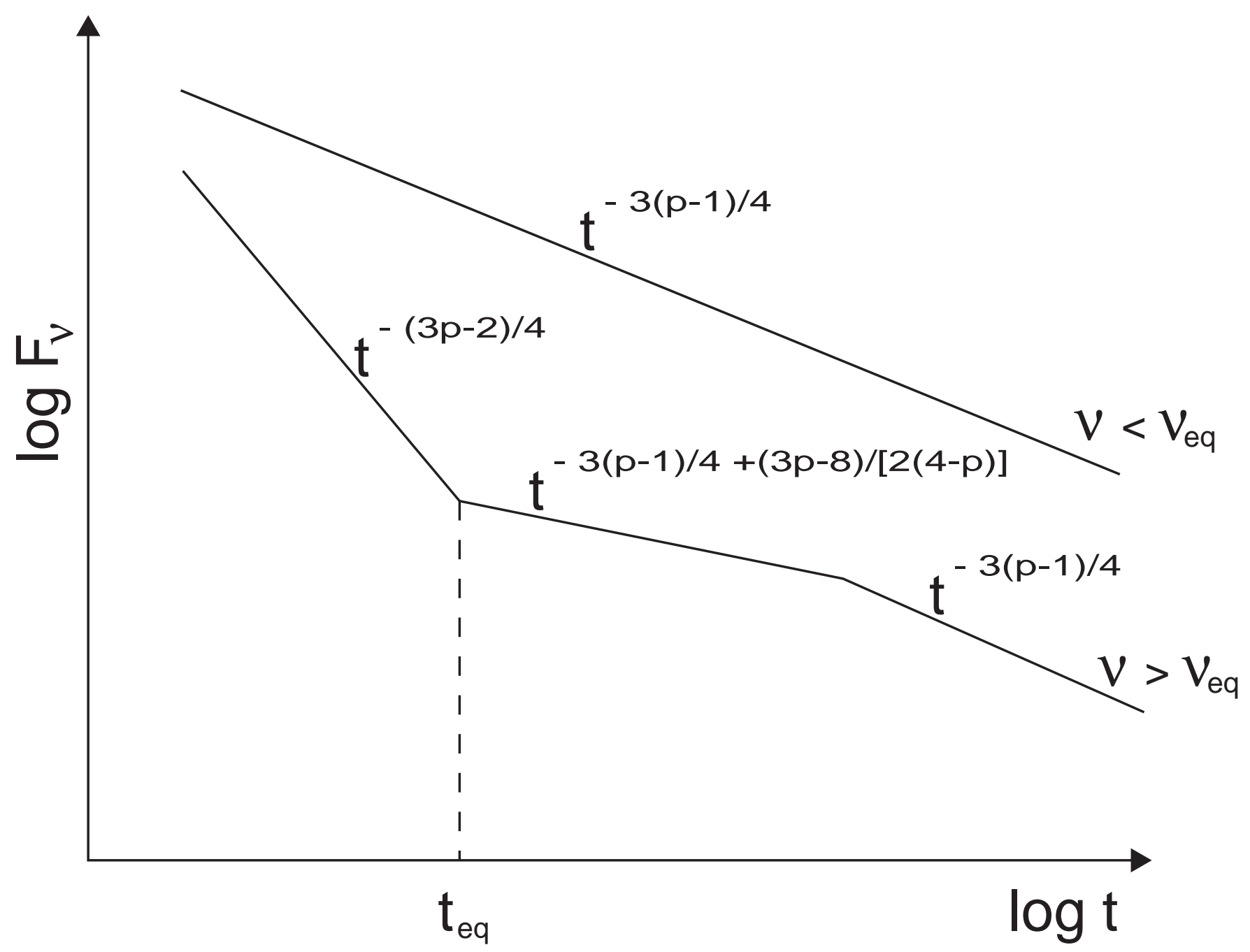

